ESAIM: COCV 27 (2021) 36

https://doi.org/10.1051/cocv/2021038
ESAIM: Control, Optimisation and Calculus of Variations

www.esaim-cocv.org

\title{
AN APPLICATION OF THE CONTINUOUS STEINER SYMMETRIZATION TO BLASCHKE-SANTALÓ DIAGRAMS*
}

\author{
Giuseppe Buttazzo** And Aldo Pratelli
}

\begin{abstract}
In this paper we consider the so-called procedure of Continuous Steiner Symmetrization, introduced by Brock in [F. Brock, Math. Nachr. 172 (1995) 25-48 and F. Brock, Proc. Indian Acad. Sci. 110 (2000) 157-204]. It transforms every open set $\Omega \subset \subset \mathbb{R}^{d}$ into the ball keeping the volume fixed and letting the first eigenvalue and the torsional rigidity respectively decrease and increase. While this does not provide, in general, a $\gamma$-continuous map $t \mapsto \Omega_{t}$, it can be slightly modified so to obtain the $\gamma$-continuity for a $\gamma$-dense class of domains $\Omega$, namely, the class of polyhedral sets in $\mathbb{R}^{d}$. This allows to obtain a sharp characterization of the Blaschke-Santaló diagram of torsion and eigenvalue.
\end{abstract}

Mathematics Subject Classification. 49Q10, 49J45, 49R05, 35P15, 35J25.

Received November 17, 2020. Accepted April 3, 2021.

\section{INTRODUCTION}

The question of making a given open set $\Omega \subset \mathbb{R}^{d}$ (through this paper we call it a domain) more and more round, keeping constant its measure, up to reach a ball, was first considered by Steiner, who proposed to use successive symmetrizations through different hyperplanes. More precisely, given a domain $\Omega \subset \mathbb{R}^{d}$ and a direction $\nu \in \mathbb{S}^{d-1}$, the Steiner symmetrization of $\Omega$ with respect to $\nu$ is defined as

$$
\Omega_{\nu}^{*}=\left\{x \in \mathbb{R}^{d}:|x \cdot \nu| \leq \frac{\varphi(\pi(x))}{2}\right\}
$$

where $\pi(x)=x-\nu(x \cdot \nu)$ is the projection of any point $x \in \mathbb{R}^{d}$ onto the hyperplane orthogonal to $\nu$ and where, for each $y$ in this hyperplane,

$$
\varphi(y)=\mathscr{H}^{1}\left(\Omega \cap \pi^{-1}(y)\right),
$$

is the length of the $y$-section of $\Omega$. The set $\Omega_{\nu}^{*}$ has the same volume of $\Omega$ and is a bit "nicer", in particular it is symmetric through the hyperplane orthogonal to $\nu$. It is not difficult to guess that, repeating this symmetrization through a sequence of hyperplanes with properly chosen directions, one obtains a sequence $\Omega_{n}$ of sets, all with the same measure, which $\gamma$-converge as $n \rightarrow \infty$ to a ball. The interest in this symmetrization procedure consists

\footnotetext{
* Dedicated to Enrique Zuazua for his 60th birthday.
}

Keywords and phrases: Blaschke-Santaló diagrams, continuous Steiner symmetrization, torsional rigidity, principal eigenvalue.

Dipartimento di Matematica, Università di Pisa Largo B. Pontecorvo 5, 56127 Pisa - Italy.

** Corresponding author: buttazzo@dm.unipi.it 
in the fact that along the sequence $\Omega_{n}$ several quantities improve, and become asymptotically optimal as $n \rightarrow \infty$. In particular we are interested in the following quantities.

- The first eigenvalue $\lambda(\Omega)$ of the Laplace operator $-\Delta$ with Dirichlet conditions on $\partial \Omega$, defined as the smallest number $\lambda$ providing a nonzero solution to the PDE

$$
-\Delta u=\lambda u \text { in } \Omega, \quad u \in H_{0}^{1}(\Omega),
$$

or equivalently through the minimization of the Rayleigh quotient

$$
\lambda(\Omega)=\min \left\{\left[\int_{\Omega}|\nabla u|^{2} \mathrm{~d} x\right]\left[\int_{\Omega}|u|^{2} \mathrm{~d} x\right]^{-1}: u \in H_{0}^{1}(\Omega) \backslash\{0\}\right\} .
$$

An important bound for $\lambda(\Omega)$ is the Faber-Krahn inequality,

$$
|\Omega|^{2 / d} \lambda(\Omega) \geq|B|^{2 / d} \lambda(B),
$$

where $B$ is any ball in $\mathbb{R}^{d}$.

- The torsional rigidity $T(\Omega)$, defined as $\int_{\Omega} u_{\Omega} \mathrm{d} x$, where $u_{\Omega}$ is the unique solution of the PDE

$$
-\Delta u=1 \text { in } \Omega \quad u \in H_{0}^{1}(\Omega),
$$

or equivalently through the maximization problem

$$
T(\Omega)=\max \left\{\left[\int_{\Omega} u \mathrm{~d} x\right]^{2}\left[\int_{\Omega}|\nabla u|^{2} \mathrm{~d} x\right]^{-1}: u \in H_{0}^{1}(\Omega) \backslash\{0\}\right\},
$$

where the maximum is reached by $u_{\Omega}$ itself. Also for $T(\Omega)$ an important inequality is true, that is, the SaintVenant inequality

$$
|\Omega|^{-(d+2) / d} T(\Omega) \leq|B|^{-(d+2) / d} T(B),
$$

where $B$ is any ball in $\mathbb{R}^{d}$.

The inequalities (1.1) and (1.2) ensure that balls minimize the first eigenvalue, and maximize the torsional rigidity, among sets of given volume. It is easy to verify that the quantities above fulfill the following scaling properties:

$$
\lambda(s \Omega)=s^{-2} \lambda(\Omega), \quad T(s \Omega)=s^{d+2} T(\Omega) .
$$

It is well-known (see for instance [1]) that the Steiner symmetrization decreases the first eigenvalue and increases the torsional rigidity, that is, for every set $\Omega \subset \mathbb{R}^{d}$ and direction $\nu \in \mathbb{S}^{d-1}$ one has

$$
\lambda\left(\Omega_{\nu}^{*}\right) \leq \lambda(\Omega), \quad T\left(\Omega_{\nu}^{*}\right) \geq T(\Omega),
$$

so that for the sequence $\Omega_{n}$ defined above one has that $\lambda\left(\Omega_{n}\right)$ (resp. $T\left(\Omega_{n}\right)$ ) decreases (resp., increases) with respect to $n$, and converges to $\lambda(B)$ (resp., $T(B)$ ), being $B$ any ball with $|B|=|\Omega|$.

A natural question is whether the discrete approximation can be replaced by a continuous one. More precisely, one would like to have a family $\Omega_{t}$, with $t \in[0,1]$, such that $\Omega_{0}=\Omega, \Omega_{1}=B$ and such that $t \mapsto \lambda\left(\Omega_{t}\right)$ and $t \mapsto T\left(\Omega_{t}\right)$ are respectively continuously decreasing and continuously increasing. In addition, the family of sets should be continuous with respect to the $\gamma$-convergence, which is the natural convergence for variational problems, and that we briefly recall in Section 2. As described above, successive Steiner symmetrizations allow to 
pass from a generic set to the ball, hence it is enough to construct a continuous approximation which transforms a set $\Omega$ into its Steiner symmetrization $\Omega_{\nu}^{*}$.

An explicit construction of a family $\Omega_{t}$ transforming the set $\Omega$ into its Steiner symmetrization $\Omega_{\nu}^{*}$, called continuous Steiner symmetrization, was proposed by Brock in [9], see also [10]. Previously, other constructions had been proposed, see for instance [6,15]. With Brock's construction, that we will briefly describe in Section 3, the quantities $\lambda\left(\Omega_{t}\right)$ and $T\left(\Omega_{t}\right)$ are respectively decreasing and increasing, but they are not continuous; more precisely, they are both continuous from the left, and respectively upper and lower semicontinuous from the right (see for instance [11]). The full $\gamma$-continuity of Brock's construction, which implies also the continuity of first eigenvalue and torsional rigidity, only holds on restricted classes of domains, as for instance the class of convex domains.

On the other hand, a $\gamma$-continuous symmetrization $\left(\Omega_{t}\right)$ which makes $\lambda\left(\Omega_{t}\right)$ and $T\left(\Omega_{t}\right)$ continuously decreasing and increasing would be very useful in several situations. In this paper we show that a simple modification of Brock's construction is enough to define such a symmetrization for the class of polyhedral domains, which are known to be $\gamma$-dense among all domains. Despite the fact that this is a very specific class, the result is enough to prove that the Blaschke-Santaló diagram corresponding to the pair $(\lambda(\Omega), T(\Omega))$ is between two graphs. Several other estimates for various kinds of quantities depending on a domain $\Omega$ are available in the recent literature; we refer the interested reader to $[2,4,8,14,16]$ and to references therein.

Let us be more precise. Calling $B$ any ball in $\mathbb{R}^{d}$, for every domain $\Omega \subset \mathbb{R}^{d}$ we define the quantities

$$
x_{\Omega}=\frac{|B|^{2 / d} \lambda(B)}{|\Omega|^{2 / d} \lambda(\Omega)}, \quad y_{\Omega}=\frac{|B|^{(d+2) / d} T(\Omega)}{|\Omega|^{(d+2) / d} T(B)},
$$

which are respectively the reciprocal of the first eigenvalue $\lambda(\Omega)$ and the torsional rigidity $T(\Omega)$, suitably rescaled so to be in the interval $[0,1]$. The Blaschke-Santaló diagram is the subset of $\mathbb{R}^{2}$ given by

$$
E=\left\{(x, y) \in \mathbb{R}^{2}: x=x_{\Omega}, y=y_{\Omega} \text { for some domain } \Omega\right\} .
$$

Since the quantities $x_{\Omega}$ and $y_{\Omega}$ above are scaling invariant, in the definition of the subset $E$ we may limit ourselves to consider only domains $\Omega$ with $|\Omega|=1$. Our two main results are then the following.

Theorem 1.1. For every polyhedron $\Omega \subset \mathbb{R}^{d}$ (not necessarily connected) there exists a $\gamma$-continuous map $[0,1] \ni t \mapsto \Omega_{t} \subset \mathbb{R}^{d}$ such that every set $\Omega_{t}$ has the same measure, $\Omega_{0}=\Omega, \Omega_{1}$ is a ball, and the quantities $t \mapsto \lambda\left(\Omega_{t}\right)$ and $t \mapsto T\left(\Omega_{t}\right)$ are respectively continuously decreasing and continuously increasing.

Theorem 1.2. There exists an increasing function $h:[0,1] \rightarrow[0,1]$ such that the Blaschke-Santaló diagram $E$ coincides with the region of $[0,1] \times[0,1]$ between the two curves

$$
y=x^{(d+2) / 2} \quad \text { and } \quad y=h(x) .
$$

More precisely,

$$
\left\{(x, y) \in[0,1]^{2}: x^{(d+2) / 2}<y<h(x)\right\} \subseteq E \subseteq\left\{(x, y) \in[0,1]^{2}: x^{(d+2) / 2} \leq y \leq h(x)\right\} .
$$

In addition, for every $x \in[0,1]$ the function $h$ satisfies

$$
x^{(d+2) / 2}\left(\left[x^{-d / 2}\right]+\left(x^{-d / 2}-\left[x^{-d / 2}\right]\right)^{(d+2) / d}\right) \leq h(x) \leq \frac{x d(d+2)^{2}}{2 x d+(d+2) \lambda(B)},
$$

where $[\cdot]$ denotes the integer part, and $B$ is a ball of radius 1. 
The approach we use to obtain Theorem 1.2 is rather general. Namely, we show that $E$ is "downward and rightward convex". More precisely, for every $\left(x_{0}, y_{0}\right) \in E$ we prove that all the points $(x, y) \in\left(x_{0}, 1\right) \times\left(0, y_{0}\right)$ with $y \geq x^{(d+2) / 2}$ belong to $E$. In the proof of this convexity property the $\gamma$-continuous Steiner symmetrization for polyhedra is crucial and the characterization of the structure of the set $E$ could be of great help in the analysis of several shape optimization problems. We briefly discuss the limit cases in the inclusions (1.3) in the final Remark 5.2.

The plan of the paper is the following. In Section 2 and in Section 3 we quickly describe the $\gamma$-convergence and the continuous Steiner symmetrization of Brock. Then, in Section 4 and in Section 5 we prove respectively Theorems 1.1 and 1.2.

\section{THE $\gamma$-CONVERGENCE}

In this section we recall the definition of $\gamma$-convergence, together with its main properties. For a more detailed analysis we refer to the book [12]. For simplicity we always assume that all the domains we consider are contained in a fixed bounded set $D \subset \mathbb{R}^{d}$, which makes no difference for our purposes.

Definition 2.1. We say that a sequence $\left\{\Omega_{n}\right\}$ of open sets $\gamma$-converges to the open set $\Omega$ if for every right-hand side $f \in H^{-1}(D)$ the solutions $u_{n}$ of the PDEs

$$
-\Delta u_{n}=f \text { in } \Omega_{n}, \quad u_{n} \in H_{0}^{1}\left(\Omega_{n}\right),
$$

each extended by zero on $D \backslash \Omega_{n}$, converge weakly in $H_{0}^{1}(D)$ to the solution $u$ of

$$
-\Delta u=f \text { in } \Omega, \quad u \in H_{0}^{1}(\Omega) .
$$

We summarize here below the main properties of the $\gamma$-convergence. We refer to [12] for all the details, properties, and proofs.

(1) The $\gamma$-convergence can be defined in a similar way for quasi-open sets $\Omega \subset D$ or more generally for capacitary measures $\mu$ confined into $D$ (that is $\mu=+\infty$ outside $D$ ). For a capacitary measure $\mu$ the corresponding PDE is written as

$$
-\Delta u+\mu u=f \text { in } D, \quad u \in H_{0}^{1}(D) \cap L_{\mu}^{2}(D),
$$

and has to be intended it in the weak sense, that is, $u \in H_{0}^{1}(D) \cap L_{\mu}^{2}(D)$ and

$$
\int_{D} \nabla u \nabla \phi \mathrm{d} x+\int_{D} u \phi \mathrm{d} \mu=\langle f, \phi\rangle \quad \forall \phi \in H_{0}^{1}(D) \cap L_{\mu}^{2}(D) .
$$

(2) The space $\mathcal{M}$ of capacitary measures above, endowed with the $\gamma$-convergence, is a compact space.

(3) Open sets or more generally quasi-open sets belong to $\mathcal{M}$; for a given domain $\Omega$ the element of $\mathcal{M}$ representing it is the measure defined for all Borel sets $E \subset D$ as

$$
\infty_{\Omega^{c}}(E)= \begin{cases}0 & \text { if } \operatorname{cap}(E \cap \Omega)=0 \\ +\infty & \text { otherwise }\end{cases}
$$

(4) In Definition 2.1 requiring the convergence of the solutions $u_{n}$ to $u$ for every right-hand side $f$ is equivalent to require the convergence $u_{n} \rightarrow u$ only for $f \equiv 1$ and in the $L^{2}(D)$ sense. In particular, calling $u_{\mu}$ the solution of the $\mathrm{PDE}-\Delta u+\mu u=1$ in $H_{0}^{1}(D) \cap L_{\mu}^{2}(D)$, the quantity

$$
d_{\gamma}\left(\mu_{1}, \mu_{2}\right)=\left\|u_{\mu_{1}}-u_{\mu_{2}}\right\|_{L^{2}(D)},
$$


is a distance on the space $\mathcal{M}$ of capacitary measures, which is equivalent to $\gamma$-convergence, and so $\mathcal{M}$ endowed with the distance $d_{\gamma}$ is a compact metric space.

Several subclasses of $\mathcal{M}$ are dense with respect to the $\gamma$-convergence (see Prop. 4.3.7 and Rem. 4.3.8 of [12]). For instance:

(i) the class of measures $a(x) \mathrm{d} x$ with $a \geq 0$ and smooth;

(ii) the class of smooth domains $\Omega \subset D$.

As a consequence of (ii) above we also have the $\gamma$-density of

(iii) the class of polyhedral domains $\Omega \subset D$.

(5) The first eigenvalue $\lambda(\Omega)$ (as well as all the other eigenvalues $\lambda_{k}(\Omega)$ ) and the torsional rigidity $T(\Omega)$ are continuous with respect to the $\gamma$-convergence.

\section{The CONTINuOUs Steiner SYMmetrizATion}

In this section we describe the continuous Steiner symmetrization studied by Brock in [9, 10]. As described in the introduction, this is a path of open sets $\Omega_{t}$ which start from a given open set $\Omega_{0}=\Omega$ and end with the Steiner symmetral $\Omega_{\infty}=\Omega_{\nu}^{*}$ of $\Omega$ with respect to a given direction $\nu \in \mathbb{S}^{d-1}$. In this construction the variable $t$ ranges from 0 to $+\infty$, while in Theorem 1.1 we preferred to use $t \in[0,1]$, this is clearly only a matter of taste and does not make any real difference.

In order to describe this symmetrization, the important issue is to discuss the one-dimensional case. Let us start assuming that $\Omega=(a, b)$ is an open segment in $\mathbb{R}$. In this case, for every $t$ the set $\Omega_{t}$ is again a segment $\left(a_{t}, b_{t}\right)$ of length $b_{t}-a_{t}=b-a$, which moves towards right with velocity $\left(b_{t}+a_{t}\right) / 2$. In other words, the position of the barycenter $m_{t}=\left(b_{t}+a_{t}\right) / 2$ is given by $e^{-t} m_{0}$, and in particular $\Omega_{\infty}=(-(a+b) / 2,(a+b) / 2)$ is the Steiner symmetral of $\Omega$.

Let us now assume that $\Omega \subset \mathbb{R}$ is given by a finite union of open segments with disjoint closures. In this case, for small $t$ each of the segments moves according with the above rule. There is then a smallest time $t_{1}>0$ when two consecutive segments meet, so in particular $\Omega_{t_{1}}$ is given by a finite union of segments, and (at least) two of them have a common endpoint. Let us call $\Omega_{t_{1}}^{+}=\operatorname{Int}\left(\overline{\Omega_{t_{1}}}\right)$, that is, we add to the set $\Omega_{t_{1}}$ the common endpoints. The set $\Omega_{t_{1}}^{+}$is then a finite union of open segments with disjoint closures, and for $t>t_{1}$ with small difference $t-t_{1}$ we can define $\Omega_{t}=\left(\Omega_{t_{1}}^{+}\right)_{t-t_{1}}$. Again, there is a smallest time $t_{2}>t_{1}$ when two consecutive segments meet, and so on. After a finite number of junctions, the set $\Omega_{t}$ is then remained a single segment, and then we leave it evolve to the symmetric segment $\Omega_{\infty}$ as already described.

As shown by Brock, there is a general rule which works for all the open subsets of $\mathbb{R}$, and which reduces to the one depicted above in the case of finitely many segments.

The construction in $\mathbb{R}^{d}$ is basically one-dimensional. Calling, for every $y \in \mathbb{R}^{d}$ orthogonal to the direction $\nu$, $\Omega^{y}$ the $y$-section of $\Omega$, made by all points $x$ of $\Omega$ such that $y-x$ is parallel to $\nu$, one simply defines $\Omega_{t}$ the set such that, for every $y,\left(\Omega_{t}\right)^{y}=\left(\Omega^{y}\right)_{t}$. As shown in [9-11,13], the family of sets $\Omega_{t}$ has various properties. They are all sets with the same measure, being $\Omega_{0}=\Omega$ and $\Omega_{\infty}=\Omega_{\nu}^{*}$. In addition, the first eigenvalue $\lambda\left(\Omega_{t}\right)$ and the torsional rigidity $T\left(\Omega_{t}\right)$ are respectively decreasing and increasing with respect to $t$. More precisely, they are both continuous from the left, and they can have jumps from the right. One can say even more, that is, if $s \quad t$ then the sets $\Omega_{s}$ are $\gamma$-converging to $\Omega_{t}$.

The reason why the sets behave badly if $s \searrow t$ can be easily understood with an example. Let us assume that $\Omega=\Omega_{0}$ has a U-shape as in Figure 1 , and that $\nu$ is the horizontal vector. The set $\Omega$ already coincides with $\Omega_{\nu}^{*}$ below a height $y_{0}$, hence for every $t>0$ the sets $\Omega_{0}, \Omega_{t}$ and $\Omega_{\infty}=\Omega_{\nu}^{*}$ coincide below this height. For a small time $\sigma>0$, the two "legs" of $\Omega$ have become closer, and they have already met below a height $y_{\sigma}$, hence below this height all the sets $\Omega_{t}$ coincide for $t>\sigma$. There is then a particular time $\tau$ when the two internal, vertical segments in the boundary of $\Omega_{\tau}$ coincide. Notice that the set $\Omega_{\tau}^{+}$defined above consists in the set $\Omega_{\tau}$ together with the internal, vertical segment, and actually $\Omega_{t}=\Omega_{\tau}^{+}=\Omega_{\infty}=\Omega_{\nu}^{*}$ for every $t>\tau$. It is obvious that the functions $t \mapsto \lambda\left(\Omega_{t}\right)$ and $t \mapsto T\left(\Omega_{t}\right)$ are continuous for $0 \leq t \leq \tau$, and according with Brock's result they are also respectively decreasing and increasing. After the time $\tau$, instead, since the vertical segment suddenly disappears, there is clearly a jump in both functions. 


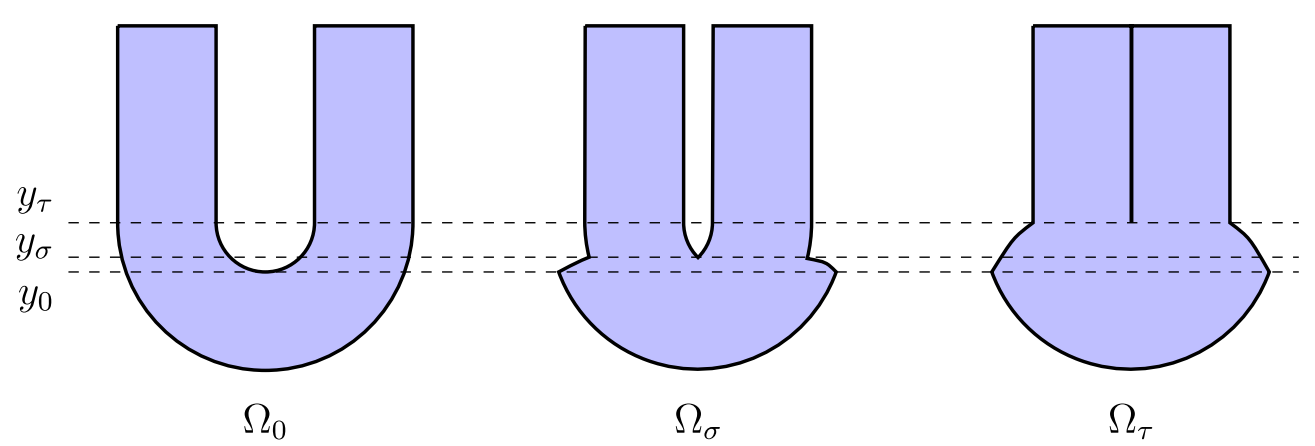

FiguRE 1 . A set $\Omega$ such that $t \mapsto \lambda\left(\Omega_{t}\right)$ is discontinuous.

\section{The CASE OF THE POLYHeDRA}

This section is devoted to consider the case of polyhedra, and to show Theorem 1.1. The idea is simple; if $\Omega$ is a polyhedron then, similarly to what happens in the example considered in Figure 1, the path $t \mapsto \Omega_{t}$ is already $\gamma$-continuous, except at finitely many instants where a $(d-1)$-dimensional wall suddenly disappears. It is then sufficient to modify the construction letting these "walls" smoothly disappear in a positive time, gaining then the $\gamma$-continuity. In the proof, the assumption that $\Omega$ is a polyhedron is crucial. We will then conclude this section with an example showing that even for smooth domains the argument does not work.

Proof (of Thm. 1.1). Let $\Omega \subset \mathbb{R}^{d}$ be a polyhedron, and let $\nu \in \mathbb{S}^{d-1}$ be a given direction. Notice that also the set $\Omega_{\nu}^{*}$ is a polyhedron. As already said in the introduction, for every open set $A$ compactly contained in $D$ we call $u_{A}$ the torsion function, i.e., the unique solution of the PDE $-\Delta u=1$ in $H_{0}^{1}$, extended by 0 in $D \backslash A$. Moreover, for every $t>0$, we define $\Omega_{t}^{+}=\operatorname{Int}\left(\overline{\Omega_{t}}\right)$.

Let us fix some $t \geq 0$. First of all, we can prove that

$$
\left|\Omega_{t}\right|=\left|\Omega_{t}^{+}\right|
$$

In fact, $\Omega_{t}$ is a polyhedron, hence for every $y$ orthogonal to $\nu$ the section $\left(\Omega_{t}\right)^{y}$ is done by a finite number of segments. As a consequence, $\overline{\left(\Omega_{t}\right)^{y}} \backslash\left(\Omega_{t}\right)^{y}$ is done by a finite number of points, thus $\mathscr{H}^{1}\left(\overline{\left(\Omega_{t}\right)^{y}}\right)=\mathscr{H}^{1}\left(\left(\Omega_{t}\right)^{y}\right)$. We can also observe that $\left(\Omega_{t}^{+}\right)^{y} \subseteq \overline{\Omega_{t}^{y}}$. In fact, if $x \notin \overline{\Omega_{t}^{y}}$, this means that some nontrivial segment centered at $x$ and parallel to $\nu$ has empty intersection with $\Omega_{t}^{y}$. And again since $\Omega_{t}$ is a polyhedron this means that a small cube centered at $x$ does not intersect $\Omega_{t}$, and so $x \notin\left(\Omega_{t}^{+}\right)^{y}$. Since of course $\Omega_{t} \subseteq \Omega_{t}^{+}$, we have then by Fubini Theorem

$$
\begin{aligned}
\left|\Omega_{t}\right| \leq\left|\Omega_{t}^{+}\right| & =\int_{y \perp \nu} \mathscr{H}^{1}\left(\left(\Omega_{t}^{+}\right)^{y}\right) d \mathscr{H}^{N-1}(y) \leq \int_{y \perp \nu} \mathscr{H}^{1}\left(\overline{\Omega_{t}^{y}}\right) d \mathscr{H}^{N-1}(y) \\
& =\int_{y \perp \nu} \mathscr{H}^{1}\left(\Omega_{t}^{y}\right) d \mathscr{H}^{N-1}(y)=\left|\Omega_{t}\right|
\end{aligned}
$$

which proves (4.1).

Let now $s_{n} \searrow t$ be a sequence converging to $t$ from above. The functions $u_{\Omega_{s_{n}}}$ form a bounded sequence in $H_{0}^{1}(D)$, hence a subsequence converges to some function $\bar{u}$ weakly in $H_{0}^{1}(D)$, so in particular strongly in $L^{2}(D)$. We claim that, since $\Omega$ is a polyhedron, then

$$
\bar{u} \in H_{0}^{1}\left(\Omega_{t}^{+}\right) .
$$


To prove this fact, we call for brevity $A=\Omega_{t}^{+}$, and we set $A_{\varepsilon}=\{x:|B(x, \varepsilon) \cap A|>0\}$ for every $\varepsilon>0$. Notice that both $A$ and $A_{\varepsilon}$ are polyhedra, and $A_{\varepsilon} \backslash A$ is a polyhedron with boundary $\partial\left(A_{\varepsilon} \backslash A\right)=\partial A_{\varepsilon} \cup \partial A$. Moreover, by construction we have that $\Omega_{s_{n}} \subseteq A_{\varepsilon}$ for every $n$ large enough, depending on $\varepsilon$. As a consequence, we have that $\bar{u} \in H_{0}^{1}\left(A_{\varepsilon}\right)$, so in particular $\bar{u}=0$ almost everywhere on $D \backslash A_{\varepsilon}$, and since this holds for every $\varepsilon>0$ then $\bar{u}=0$ a.e. on $D \backslash \cap_{\varepsilon>0} A_{\varepsilon}$. Again minding that $A$ is a polyhedron, the set $D \backslash \cap_{\varepsilon>0} A_{\varepsilon}$ coincides with $D \backslash \bar{A}$, hence with $D \backslash A$ up to a set of null measure. In other words, $\bar{u} \in H_{0}^{1}(D)$ is a function which equals 0 almost everywhere on $D \backslash A$. Notice carefully that this does not imply $\bar{u} \in H_{0}^{1}(A)$, for a general open set. Nevertheless, as noticed above $A_{\varepsilon} \backslash A$ is a polyhedron with boundary $\partial A_{\varepsilon} \cup \partial A$. The fact that $\bar{u}=0$ almost everywhere in $A_{\varepsilon} \backslash A$ implies that the trace of $u$ is zero on $\partial\left(A_{\varepsilon} \backslash A\right)$, hence in particular on $\partial A$. And finally, this means $\bar{u} \in H_{0}^{1}(A)$, which is (4.2). This inclusion is crucial, and it is false in general if $\Omega$ is not a polyhedron, even being smooth does not suffice, see Example 4.1. This is the reason why Theorem 1.1 is stated for polyhedra.

Notice now that by $(4.2)$

$$
T\left(\Omega_{t}^{+}\right) \geq \frac{\left(\int \bar{u} \mathrm{~d} x\right)^{2}}{\int|\nabla \bar{u}|^{2} \mathrm{~d} x} \geq \limsup _{n \rightarrow \infty} \frac{\left(\int u_{n} \mathrm{~d} x\right)^{2}}{\int\left|\nabla u_{n}\right|^{2} \mathrm{~d} x}=\limsup _{n \rightarrow \infty} T\left(\Omega_{n}\right) \geq T\left(\Omega_{t}^{+}\right) .
$$

The last inequality is true because the torsional rigidity increases with time and, by definition, for every $s>t$ one has that $\Omega_{s}=\left(\Omega_{t}\right)_{s-t}=\left(\Omega_{t}^{+}\right)_{s-t}$. By the above chain of inequalities, and by the uniqueness of the torsion function, we deduce that $\bar{u}=u_{\Omega_{t}^{+}}$. Therefore, since the convergence of $u_{\Omega_{s_{n}}}$ to $u_{\Omega}=\bar{u}$ is strong also in $L^{1}$, we deduce that the sets $\Omega_{s}$, when $s \searrow t, \gamma$-converge to $\Omega_{t}^{+}$.

Observe now that, by construction, $\Omega_{t}$ is an open set contained in $\Omega_{t}^{+}$, and they have the same measure by (4.1). By the maximum principle we have $u_{\Omega_{t}} \leq u_{\Omega_{t}^{+}}$, thus

$$
d_{\gamma}\left(\Omega_{t}, \Omega_{t}^{+}\right)=\left\|u_{\Omega_{t}}-u_{\Omega_{t}^{+}}\right\|_{L^{1}}=\int\left(u_{\Omega_{t}^{+}}-u_{\Omega_{t}}\right) \mathrm{d} x=T\left(\Omega_{t}^{+}\right)-T\left(\Omega_{t}\right)
$$

Again using the fact that $\Omega$ is a polyhedron, there can be at most finitely many instants $t_{1}<t_{2}<\cdots<$ $t_{N}$ such that the above difference is strictly positive, thus the path $t \mapsto \Omega_{t}$ is already $\gamma$-continuous in $\mathbb{R}_{+} \backslash$ $\left\{t_{1}, t_{2}, \ldots, t_{N}\right\}$.

Let now $t$ be any of the instants $t_{j}$. We aim to define an increasing family of sets $\Omega_{t, \eta}$, for $0 \leq \eta \leq 1$, ranging from $\Omega_{t, 0}=\Omega_{t}$ to $\Omega_{t, 1}=\Omega_{t}^{+}$, in a $\gamma$-continuous way. The idea is simple, and it is already contained in [11]. We define a large cube $Q$, with sides parallel to the coordinate axes and side $\ell$, containing $D$. We can then split $Q$ as the essentially disjoint union of $2^{d}$ cubes $Q_{j}$, for $1 \leq j \leq 2^{d}$, each with side $\ell / 2$. Analogously, each cube $Q_{j}$ is the union of cubes $Q_{j, h}$ with $1 \leq h \leq 2^{d}$ and side $\ell / 4$, and so on. For every finite sequence $J=\left\{j_{1}, j_{2}, \ldots, j_{m}\right\}$ of length $m$ in $\left\{1,2, \ldots, 2^{d}\right\}$, we call

$$
\eta_{J}=\sum_{i=1}^{m-1}\left(j_{i}-1\right) 2^{-i d}+j_{m} 2^{-m d} \in(0,1),
$$

and we call $\operatorname{Seq}(d)$ the set of those finite sequences, which is a dense subset of $[0,1]$. For every $0 \leq \eta \leq 1$, we call

$$
Q(\eta)=\bigcup\left\{Q_{J}: J \in S e q(d), \eta_{J}<\eta\right\}
$$

so that $\eta \mapsto Q(\eta)$ is an increasing family of sets ranging from $Q(0)=\emptyset$ to $Q(1)=Q$. Finally, we define $\Omega_{t, \eta}=$ $\Omega_{t} \cup\left(\Omega_{t}^{+} \cap Q(\eta)\right)$, so that $\eta \mapsto \Omega_{t, \eta}$ is an increasing family of sets starting with $\Omega_{t, 0}=\Omega_{t}$ and ending with 


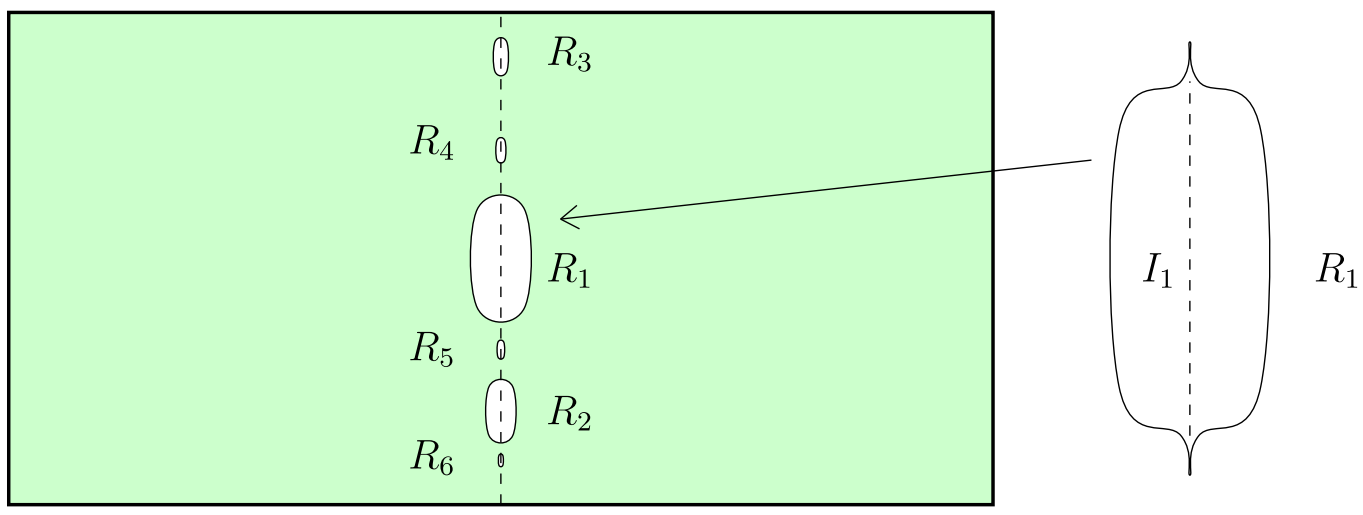

Figure 2. The set of Example 4.1 and a zoom of $R_{1}$ showing that it meets $I_{1}$ tangentially.

$\Omega_{t, 1}=\Omega_{t}^{+}$. All the sets have the same volume by (4.1), and since the sets are increasing with $\eta$ then the first eigenvalue and the torsional rigidity are respectively decreasing and increasing. Finally, the map $\eta \mapsto \Omega_{t, \eta}$ is $\gamma$-continuous by construction, since the capacity of the cubes goes to 0 when the side goes to 0 .

It is now clear how to modify the definition of the sets $\Omega_{t}$, replacing every instant $\left\{t_{j}\right\}$ with a closed time interval of width 1 , in such a way the map $[0,+\infty] \ni t \mapsto \Omega_{t}$ is a $\gamma$-continuous path between $\Omega$ and $\Omega_{\nu}^{*}$ and the first eigenvalue and the torsional rigidity are monotone (respectively decreasing and increasing) and continuous.

It is then sufficient to perform the same construction countably many times in different directions, so to eventually obtain a family of sets that $\gamma$-converge to a ball. By reparametrizing the variable $t$, we can let it vary in the closed interval $[0,1]$.

A quick look to the proof makes it clear that the assumption that $\Omega$ is a polyhedron is crucial at many steps. In particular, having defined $A=\Omega_{t}^{+}$, we have observed that the function $\bar{u}$ belongs to $H_{0}^{1}(D)$ and is 0 almost everywhere outside of $A$, and we have deduced that $\bar{u} \in H_{0}^{1}(A)$. For a domain $A$ with the additional property that $A=\operatorname{Int}(\bar{A})$, this is in general false, even if $A$ is smooth. Instead, as we have observed, this is true for a polyhedron. Keep in mind that the inclusion $\bar{u} \in H_{0}^{1}(A)$ was essential to obtain that $\lambda\left(\Omega_{t}^{+}\right)=\lim _{s \backslash t} \lambda\left(\Omega_{s}\right)$, and without this equality the whole construction cannot work. In other words, for general domains, even smooth, we do not have the guarantee that the above equality holds true. We can now present an example to show that the strict inequality may actually occur.

Example 4.1. We build a simple situation of a regular domain $\Omega$ such that for some $t \geq 0$ the inequality $\lambda\left(\Omega_{t}^{+}\right)>\lim _{s \backslash t} \lambda\left(\Omega_{s}\right)$ occurs. For simplicity we work with a planar domain, but it is simple to modify the example for any dimension $d \geq 3$. As depicted in Figure 2, we remove from the open rectangle $(-1,1) \times(0,1)$ countably many closed "curved rectangles" $R_{i}, i \in \mathbb{N}$ and the whole segment $S=\{0\} \times(0,1)$. As in the figure, each rectangle is symmetric with respect to the segment $S$, and it is much thinner than high. Moreover, we call $I_{i}=R_{i} \cap S$. Notice that this is an open set, and it is possible to find a smooth domain $\Omega$ and some $t>0$ such that, having taken the direction $\nu=(1,0)$, this set coincides with $\Omega_{t}$ (in fact, the "curved rectangles" $R_{i}$ have to meet the intervals $I_{i}$ tangentially, as the zoom in the figure explains).

We can select the intervals $I_{i}$ in such a way that they are dense in $S$, but their total length is much less than the length of $S$. In particular, the capacity of the union of the sets $R_{i}$ can be made very small. By definition of the continuous Steiner symmetrization, each hole $R_{i}$ is shrunk after the time $t$, and it is completely filled at some $s>t$. In particular, for each $s>t$ only finitely many holes $R_{i}$ are not completely filled in $\Omega_{s}$. In other words, $\Omega_{s}$ looks more or less as $\Omega_{t}$, with the crucial difference that it has only finitely many holes, and more importantly that it contains most of the segment $S$ (only the part of $S$ contained in the surviving rectangles is not contained in $\Omega_{s}$ ). Therefore, since the capacity of the surviving holes is less than the capacity of the complete union of the $R_{i}$, which is small, then $\lambda\left(\Omega_{s}\right)$ is only slightly larger than the first eigenvalue of the whole 
rectangle $(-1,1) \times(0,1)$. On the other hand, $\Omega_{t}$ coincides with $\Omega_{t}^{+}$, because since the intervals $I_{i}$ are dense in $S$ then no point of $S$ can be contained in $\Omega_{t}^{+}$. In particular, $\Omega_{t}^{+}$is contained in $(-1,1) \times(0,1) \backslash S$, thus its first eigenvalue is larger than $\lambda((-1,1) \times(0,1) \backslash S)=\lambda((0,1) \times(0,1))$, which is much bigger than $\lambda\left(\Omega_{s}\right)$ for each $s>t$.

\section{Application to the Blaschke-Santaló diagram}

The study of Blaschke-Santaló diagrams is a very powerful way to treat shape optimization problems, which are in general rather difficult to attack because the class of admissible shapes do not have strong functional properties and very often limits of sequences of shapes (in particular $\gamma$-limits) are not shapes any more. If $A(\Omega)$

and $B(\Omega)$ are two shape functionals (a similar argument can be used for a larger number of them) many shape optimization problems can be written in the form

$$
\min \{F(A(\Omega), B(\Omega)):|\Omega|=m\}
$$

where the Lebesgue measure constraint is very natural in this kind of problems. Sometimes, the presence of additional geometric constraints (as for instance convexity of admissible shapes or other geometric bounds a priori imposed) makes the above problem easier, since extra compactness properties can be deduced. When the quantities $A(\Omega)$ and $B(\Omega)$ fulfill suitable scaling relations as

$$
A(t \Omega)=t^{\alpha} A(\Omega), \quad B(t \Omega)=t^{\beta} B(\Omega),
$$

and if the function $F$ is expressed through powers, as

$$
F(A, B)=A^{p} B^{q}
$$

the Lebesgue measure constraint $|\Omega|=m$ can be incorporated in the scaling free functional

$$
\mathcal{F}(\Omega)=\frac{A^{p}(\Omega) B^{q}(\Omega)}{|\Omega|^{(\alpha p+\beta q) / d}}=\left(\frac{A(\Omega)}{|\Omega|^{\alpha / d}}\right)^{p}\left(\frac{B(\Omega)}{|\Omega|^{\beta / d}}\right)^{q},
$$

and the minimum problem above can be reformulated as the minimum problem for $\mathcal{F}$ without any Lebesgue measure constraint.

The Blaschke-Santaló diagram for the pair $A(\Omega), B(\Omega)$ is the subset of the Euclidean space $\mathbb{R}^{2}$ given by

$$
E=\left\{(x, y) \in \mathbb{R}^{2}: x=\frac{A(\Omega)}{|\Omega|^{\alpha / d}}, y=\frac{B(\Omega)}{|\Omega|^{\beta / d}} \text { for some } \Omega\right\} .
$$

In this way our shape optimization problem (5.1) can be reduced to the optimization problem on $\mathbb{R}^{2}$ given by

$$
\min \{F(x, y):(x, y) \in E\} .
$$

In general the full characterization of the Blaschke-Santaló diagram $E$ is a difficult problem and often only some bounds can be obtained. In the present paper we consider the quantities $\lambda(\Omega)$ and $T(\Omega)$ and we try to identify the set $E$ in this case. In order to have the set $E$ included in the square $[0,1] \times[0,1]$ it is convenient to take the rescaled variables

$$
x=\frac{|B|^{2 / d} \lambda(B)}{|\Omega|^{2 / d} \lambda(\Omega)}, \quad \quad y=\frac{|B|^{(d+2) / d} T(\Omega)}{|\Omega|^{(d+2) / d} T(B)},
$$




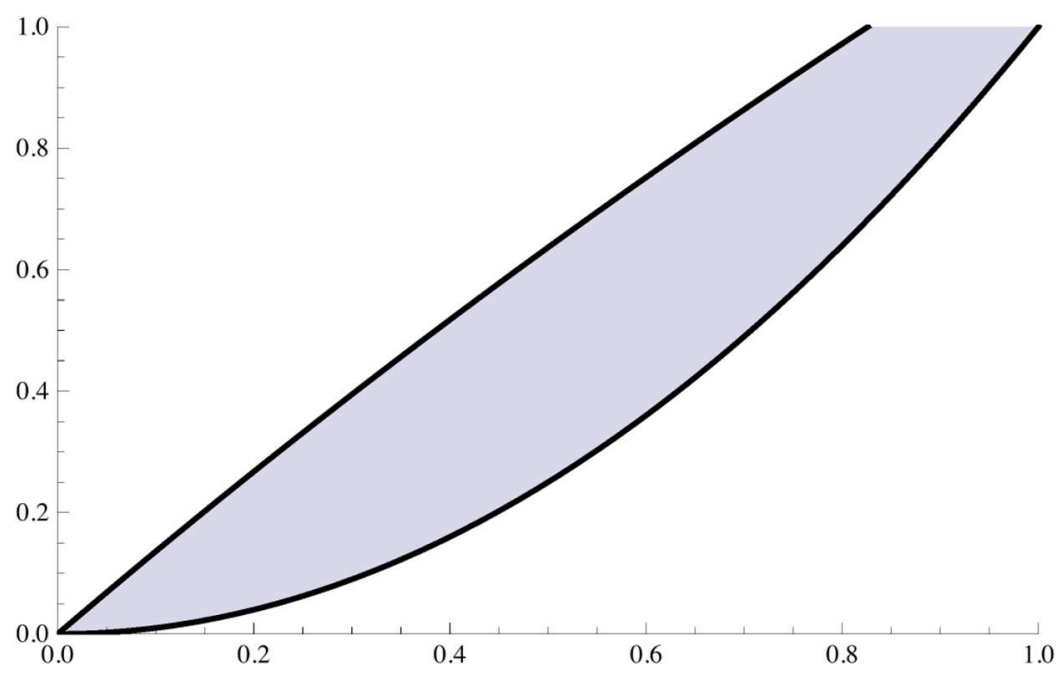

Figure 3. The colored region, obtained by the inequalities (5.4) and (5.5), contains the Blaschke-Santaló diagram $E$ for $\lambda(\Omega)$ and $T(\Omega)$ in the case $d=2$.

being $B$ a ball of radius 1 . In this way the Kohler-Jobin inequality (see for instance [3])

$$
\lambda(\Omega) T^{2 /(d+2)}(\Omega) \geq \lambda(B) T^{2 /(d+2)}(B),
$$

becomes, in the $x, y$ variables,

$$
y \geq x^{(d+2) / 2} .
$$

Instead, the Polya inequality $\lambda(\Omega) T(\Omega)<|\Omega|$ (see [3]) becomes

$$
y<\frac{|B|}{\lambda(B) T(B)} x .
$$

A slight improvement of this inequality has been obtained in [5], where it is proved that

$$
\lambda(\Omega) T(\Omega) \leq|\Omega|\left(1-\frac{2 d|B|^{2 / d}}{d+2} \frac{T(\Omega)}{|\Omega|^{(d+2) / d}}\right)
$$

which, by (5.2) and since a simple calculation ensures $T(B)=\omega_{d} /(d(d+2))$, gives

$$
y \leq \frac{|B| x}{\lambda(B) T(B)}\left(1-\frac{2 x d}{2 x d+(d+2) \lambda(B)}\right) .
$$

In Figure 3 we plot the bounds (5.4) and (5.5) in the case of dimension two, which are

$$
x^{2} \leq y \leq \frac{8 x}{x+j_{0}^{2}},
$$

being $j_{0}=2.4048 \ldots$ the first zero of the Bessel function $J_{0}$.

We start to study some properties of the Blaschke-Santaló diagram $E$. 
Lemma 5.1. For every $\left(x_{0}, y_{0}\right) \in E$ there exists a sequence of continuous curves $\left(x_{n}(\sigma), y_{n}(\sigma)\right)$ in $E$, with $\sigma \in[0,1]$, such that $\left(x_{n}(0), y_{n}(0)\right)=\left(x_{0}, y_{0}\right)$, converging uniformly to the curve

$$
x(\sigma)=(1-\sigma)^{2} x_{0}, \quad y(\sigma)=(1-\sigma)^{d+2} y_{0}, \quad \sigma \in[0,1]
$$

which connects the point $\left(x_{0}, y_{0}\right)$ with the origin. In Cartesian coordinates the limit curve is the graph of the function

$$
y=y_{0}\left(x / x_{0}\right)^{(d+2) / 2} \quad x \in\left[0, x_{0}\right] .
$$

Proof. Let $\Omega$ be a domain which gives the point $\left(x_{0}, y_{0}\right) \in E$, that is

$$
x_{0}=\frac{|B|^{2 / d} \lambda(B)}{|\Omega|^{2 / d} \lambda(\Omega)}, \quad \quad y_{0}=\frac{|B|^{(d+2) / d} T(\Omega)}{|\Omega|^{(d+2) / d} T(B)} .
$$

For every $n$ let $a_{n}=1-n^{-1 / d}$ and, for $\sigma \in[0,1]$, let $\Omega_{\sigma}^{n}$ be the domain which consists of the union of $\left(1-a_{n} \sigma\right) \Omega$ and $n-1$ disjoint copies of $\left(\frac{1-\left(1-a_{n} \sigma\right)^{d}}{n-1}\right)^{1 / d} \Omega$. We have $\left|\Omega_{\sigma}^{n}\right|=|\Omega|$ and

$$
\left\{\begin{array}{l}
\lambda\left(\Omega_{\sigma}^{n}\right)=\left(1-a_{n} \sigma\right)^{-2} \lambda(\Omega) \\
T\left(\Omega_{\sigma}^{n}\right)=\left[\left(1-a_{n} \sigma\right)^{d+2}+(n-1)^{-2 / d}\left(1-\left(1-a_{n} \sigma\right)^{d}\right)^{(d+2) / d}\right] T(\Omega) .
\end{array}\right.
$$

In terms of $(x, y)$ variables we have the curve

$$
\left\{\begin{array}{l}
x_{n}(\sigma)=x_{0}\left(1-a_{n} \sigma\right)^{2} \\
y_{n}(\sigma)=y_{0}\left[\left(1-a_{n} \sigma\right)^{d+2}+(n-1)^{-2 / d}\left(1-\left(1-a_{n} \sigma\right)^{d}\right)^{(d+2) / d}\right]
\end{array} \quad \sigma \in[0,1],\right.
$$

or, in Cartesian coordinates,

$$
y=y_{0}\left[\left(x / x_{0}\right)^{(d+2) / 2}+(n-1)^{-2 / d}\left(1-\left(x / x_{0}\right)^{d / 2}\right)^{(d+2) / d}\right] \quad x / x_{0} \in\left[\left(1-a_{n}\right)^{2}, 1\right] .
$$

It is immediate to see the uniform convergence of the sequence of curves $\left(x_{n}(\sigma), y_{n}(\sigma)\right)$ to the limit curve

$$
x(\sigma)=(1-\sigma)^{2} x_{0}, \quad y(\sigma)=(1-\sigma)^{d+2} y_{0}, \quad \sigma \in[0,1],
$$

as required.

We are now in a position to prove our result concerning the structure of the Blaschke-Santaló diagram $E$ of all points $(x, y) \in \mathbb{R}^{2}$ with $x$ and $y$ given by (5.2).

Proof (of Thm. 1.2). In order to prove the existence of an increasing function $h$ satisfying (1.3) it is enough to show that, for every $\left(x_{0}, y_{0}\right) \in E$, all the points $(x, y) \in[0,1]^{2}$ with $y>x^{(d+2) / 2}$ and with $x>x_{0}, y<y_{0}$ are also contained in $E$. To obtain this convexity property we rely on Theorem 1.1 and Lemma 5.1. More precisely, let $\left(x_{0}, y_{0}\right) \in E$, and let us first assume that it corresponds via (5.2) to a polyhedron $\Omega$. Let then $\Omega_{t}$, with $t \in[0,1]$, be the $\gamma$-continuous map given by Theorem 1.1, and let $\varphi:[0,1] \rightarrow E$ be the map given by $\varphi(t)=\left(x_{t}, y_{t}\right)$, where $\left(x_{t}, y_{t}\right)$ is given by (5.2) with $\Omega_{t}$ in place of $\Omega$. By Theorem 1.1, $\varphi$ is a curve which continuously connects $\left(x_{0}, y_{0}\right)$ with $(1,1)$, and which is increasing in both variables. For every $0 \leq t \leq 1$, by Lemma 5.1 we have a sequence $\xi_{n, t}$ of continuous curves, explicitely given by (5.6) and contained in $E$, all starting from $\left(x_{t}, y_{t}\right)$ and uniformly 
$(0,1)$

$(0,0)$

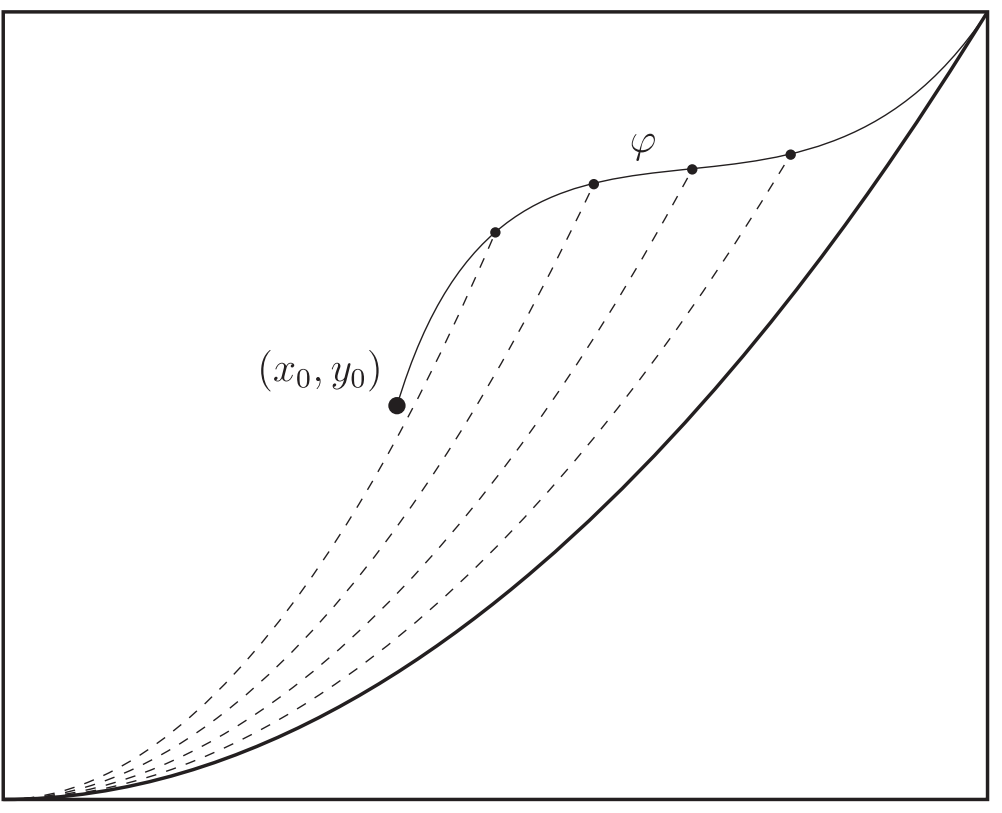

FiguRE 4. Argument of the proof of Theorem 1.2.

converging, for $n \rightarrow \infty$, to the function $\xi_{\infty, t}(x)=y_{t}\left(x / x_{t}\right)^{(d+2) / 2}, x \in\left[0, x_{t}\right]$. A simple continuity argument, graphically depicted in Figure 4, implies then that all the points $(x, y)$ with $x_{0}<x<1$ and $x^{(d+2) / 2}<y<y_{0}$ belong to $E$. More precisely, let us fix any pair $(x, y)$ with $x_{0}<x<1$ and $x^{(d+2) / 2}<y<y_{0}$. Since all the curves $\xi_{n, t}$ uniformly converge to the curves $\xi_{\infty, 0}$, there exists some large $n$ with the property that all the points $\xi_{n, t}(1)$ have second coordinate strictly smaller than $y$. Moreover, the second coordinate of the point $\xi_{n, t}(1)$ is $y_{t}>t$. Therefore, the graph of $\xi_{n, t}$ has a single point with second coordinate equal to $y$, and the first coordinate of such point can be either strictly smaller than $x$, or strictly larger, or equal (we will briefly say that the graph $\xi_{n, t}$ passes respectively on the left, or on the right, or through $\left.(x, y)\right)$. Since the graph of $\xi_{\infty, 0}$ passes on the left of $(x, y)$ and the graph of $\xi_{\infty, 1}$ passes on the right, and since the curves $\xi_{n, 0}$ and $\xi_{n, 1}$ uniformly converge to $\xi_{\infty, 0}$ and $\xi_{\infty, 1}$, then up to further increase $n$ we can assume that the graphs of $\xi_{n, 0}$ and $\xi_{n, 1}$ respectively pass on the left and on the right of the point $(x, y)$. We fix then such $n$, and we consider the curves $\xi_{n, t}$ for $t \in[0,1]$. By continuity in the $t$ variable, there exists some $\bar{t} \in(0,1)$ such that the graph of $\xi_{n, \bar{t}}$ passes through $(x, t)$. And in turn, since such graph is contained in $E$ then we have $(x, y) \in E$ as stated.

Let us now take a generic point $\left(x_{0}, y_{0}\right) \in E$, corresponding to an open domain $\Omega$. Let $\left\{\Omega_{k}\right\}_{k \in \mathbb{N}}$ be a sequence of polyhedra which approximate $\Omega$ from inside, hence which $\gamma$-converge to $\Omega$. Let us also call $\left(x_{k}, y_{k}\right)$ the numbers given by (5.2) with $\Omega_{k}$ in place of $\Omega$, so that the points $\left(x_{k}, y_{k}\right)$ converge to $\left(x_{0}, y_{0}\right)$. Let again $(x, y)$ be a pair with $x_{0}<x<1$ and $x^{(d+2) / 2}<y<y_{0}$, and let us prove that $(x, y) \in E$. The argument already presented for polyhedra ensures that this is true if $x>x_{k}$ and $y<y_{k}$ for some $k \in \mathbb{N}$, and in turn this is obviously true for $k$ large enough since $\left(x_{k}, y_{k}\right) \rightarrow\left(x_{0}, y_{0}\right)$.

As already noticed at the beginning, this shows the existence of an increasing function $h$ satisfying (1.3) follows. Finally, concerning the bound (1.4) on $h$, the upper one coincides with (5.5), and the lower one is proved in ([3], Prop. 7.2).

Remark 5.2. We conclude with a short discussion about the equalities in (1.3). More precisely, it would be interesting to determine whether or not the points $(x, y) \in[0,1]^{2}$ with $y=x^{(d+2) / 2}$ or with $y=h(x)$ belong 
to $E$. The first part is actually known. Indeed, as observed in [7, Rem. 4.2], the Kohler-Jobin inequality (5.3) is strict for every set $\Omega$ which is not a ball. Therefore, the point $\left(x, x^{(d+2) / 2}\right)$ does not belong to $E$ for every $0 \leq x<1$, while of course $(1,1) \in E$, since it corresponds to the ball. Instead, we do not know whether the points $(x, h(x))$ belong to $E$ for $0<x<1$.

Remark 5.3. Along all the paper we considered as domains $\Omega$ the open subsets of $\mathbb{R}^{d}$. Thanks to the $\gamma$-density of the class of polyhedral sets seen in Section 2, the statement of Theorem 1.2 would have been the same if the Blaschke-Santaló diagram $E$ was defined by means of the larger class of quasi-open sets (i.e. positivity sets of $H^{1}\left(\mathbb{R}^{d}\right)$ functions), or even more generally by means of capacitary measures of the class $\mathcal{M}$.

Remark 5.4. It seems reasonable to guess that $h$ is one-to-one, or equivalently that it is continuous and strictly increasing. However, we are not aware of an argument which could prove this statement. Much more importantly, it seems reasonable to guess that the claim of Theorem 1.1 should be valid for every open set, not just for polyhedra. Such a result would be extremely interesting, but it seems that our argument cannot be applied to sets which are not polyhedra, even if they are smooth. The main reason is that, even for a smooth set, a bad behaviour as the one depicted in Example 4.1 could in principle happen for all the directions $\nu$, and this would probably force to find a completely different argument, not relying on Brock's construction as starting brick.

Acknowledgements. This work is part of the project 2017TEXA3H "Gradient flows, Optimal Transport and Metric Measure Structures" funded by the Italian Ministry of Research and University. The authors are member of the Gruppo Nazionale per l'Analisi Matematica, la Probabilità e le loro Applicazioni (GNAMPA) of the Istituto Nazionale di Alta Matematica (INdAM).

\section{REFERENCES}

[1] A. Alvino, P.L. Lions and G. Trombetti, Comparison results for elliptic and parabolic equations via symmetrization: a new approach. Differ. Integral Equations 4 (1991) 25-50.

[2] M. van den Berg and G. Buttazzo, On capacity and torsional rigidity. Bull. Lond. Math. Soc. 53 (2021) 347-359.

[3] M. van den Berg, G. Buttazzo and A. Pratelli, On the relations between principal eigenvalue and torsional rigidity. To appear in: Commun. Contemp. Math. (2020). https://doi.org/10.1142/S0219199720500935

[4] M. van den Berg, G. Buttazzo and B. Velichkov, Optimization problems involving the first Dirichlet eigenvalue and the torsional rigidity, in New Trends in Shape Optimization. Birkhäuser Verlag, Basel (2015) 19-41.

[5] M. van den Berg, V. Ferone, C. Nitsch and C. Trombetti, On Pólya's inequality for torsional rigidity and first Dirichlet eigenvalue. Integral Equations Oper. Theory 86 (2016) 579-600.

[6] H.J. Brascamp, H. Lieb and J.M. Luttinger, general rearrangement inequality for multiple integrals. J. Funct. Anal. 17 (1974) $227-237$.

[7] L. Brasco, On torsional rigidity and principal frequencies: an invitation to the Kohler-Jobin rearrangement technique. $C O C V$ 20 (2014) 315-338.

[8] L. Briani, G. Buttazzo and F. Prinari, Some inequalities involving perimeter and torsional rigidity. To appear in: Appl. Math. Optim. (2020). https://doi.org/10.1007/s00245-020-09727-7

[9] F. Brock, Continuous Steiner-symmetrization. Math. Nachr. 172 (1995) 25-48.

[10] F. Brock, Continuous rearrangement and symmetry of solutions of elliptic problems. Proc. Indian Acad. Sci. 110 (2000) 157-204.

[11] D. Bucur, G. Buttazzo and I. Figueiredo, On the attainable eigenvalues of the Laplace operator. SIAM J. Math. Anal. 30 (1999) 527-536.

[12] D. Bucur and G. Buttazzo, Variational methods in shape optimization problems. Prog. Nonlinear Differ. Equations 65 (2005).

[13] D. Bucur and A. Henrot, Stability for the Dirichlet problem under continuous steiner symmetrization. Potential Anal. 13 (2000) $127-145$.

[14] I. Ftouhi and J. Lamboley, Blaschke-Santaló diagram for volume, perimeter, and first Dirichlet eigenvalue. Preprint https: //hal.archives-ouvertes.fr.

[15] B. Kawohl, Rearrangements and convexity of level sets in PDE. Springer Lecture notes Math. 1150 (1985) 7-44.

[16] I. Lucardesi and D. Zucco, On Blaschke-Santaló diagrams for the torsional rigidity and the first Dirichlet eigenvalue. Preprint arxiv 1910.04454. 